\title{
La evolución de la propiedad y la riqueza catastral de Castilla-La Mancha y su relación con España
}

\author{
FÉLIX Pillet CAPDEPÓN \\ Departamento de Geografía y Ordenación del Territorio \\ Universidad de Castilla-La Mancha \\ felix.pillet@uclm.es
}

Recibido: 19 de Febrero de 2009

Enviado a evaluar: 2 de Marzo de 2009

Aceptado: 24 de Febrero de 2011

\section{RESUMEN}

El estudio de la valoración catastral, tanto de Rústica como de Urbana, se realiza para Castilla-La Mancha comparándola con la media nacional, y como consecuencia de un proceso de análisis de la evolución de la propiedad desde el Catastro de Ensenada hasta las estadísticas del actual Catastro Inmobiliario, en sus distintas escalas, especialmente en la supramunicipal o comarcal. En el contexto español, esta Comunidad Autónoma destaca en concentración de la propiedad rústica, mientras queda muy reducida su presencia en la riqueza catastral urbana.

Palabras clave: propiedad, riqueza catastral rústica y urbana, Castilla-La Mancha y España

\section{The evolution of property and the complexity of the land registry in Castile-La Mancha and its relation to Spain}

\begin{abstract}
The study of Castile-La Mancha's land registry appraisals for both Rural and Urban properties is being carried out, with reference to the national average. It has arisen out of an analysis of how property has evolved from the time of the Ensenada land registry to the current real estate land registry statistics on their different scales, particularly at supramunicipal or regional levels. Within this autonomous region, the high concentration of country estates stands out compared to Spain as a whole while the complexity of the urban land register remains low.
\end{abstract}

Key words: property, complex rural and urban land registry, Castile-La Mancha, Spain 


\title{
Évolution de la propriété et de la valeur cadastrale de la communauté autonome de Castille-La Manche et mise en parallèle avec l'Espagne
}

\begin{abstract}
RÉSUMÉ
Pour étudier la valeur cadastrale de la communauté autonome de Castille-La Manche, en zone rurale comme en zone urbaine, il convient de la rapprocher de la moyenne nationale. Cette étude s'inscrit dans le cadre d'un processus d'analyse de l'évolution de la propriété, du Cadastre de Ensenada aux statistiques de l'actuel cadastre immobilier, et ce à différents niveaux, particulièrement supracommunal ou régional. Sous un angle national, cette communauté autonome se distingue par la concentration des propriétés en zone rurale alors que sa valeur cadastrale urbaine reste très réduite.
\end{abstract}

Mots-clés: propriété, valeur cadastrale en zone rurale et urbaine, Castille-La Manche, Espagne

\section{INTRODUCCIÓN}

Los estudios sobre la propiedad rústica y urbana han sido tratados de una forma desigual, de hecho Tatjer (1988) advertía que las investigaciones sobre la propiedad urbana fueron muy escasas, justificando esta situación en que mientras la tierra era considerada como un elemento productivo de primer orden, los inmuebles urbanos eran tenidos en cuenta como de menor importancia económica y por lo tanto fiscal. Por el contrario, las realizadas sobre la propiedad rústica han producido un monumental esfuerzo de tratamiento de fuentes de distinta naturaleza, desde un enfoque diacrónico (Mata y Naranjo, 1996). En los últimos años, Mas volvía a insistir en esta circunstancia, cuando indicaba que parecía "como si otras consideraciones hubieran suplido al análisis de la propiedad urbana" (Mas, 2005: 242).

Se podría decir que el interés por el estudio del catastro y de la propiedad, en general, ha descendido de forma importante, olvidando el pasado interés por su análisis (Pillet, 2009) como elemento fundamental de la ordenación del territorio. Tal vez la razón podamos encontrarla en la Ley Orgánica de Protección de Datos de Carácter Personal (1999), aunque la misma hace una salvedad para "el tratamiento posterior de los datos con fines históricos, estadísticos o científicos" (artículo 21-1). La excepción destacada sobre esta temática durante el presente siglo, se concreta en las publicaciones de Francisco Feo sobre la propiedad rústica desde una perspectiva de género (Feo, 2001, 2003, 2005, 2008), aspecto de gran interés y actualidad.

La crisis que estamos viviendo debe buscar soluciones en la Planificación: territorial, urbanística y estratégica y todas ellas relacionarlas con la valoración catastral, tal y como hemos defendido recientemente (Pillet, 2010 a). El Catastro Inmobiliario actual nos ha permitido desde sus bases estadísticas ofrecidas por la Dirección General del Catastro poder reflejar una serie de conclusiones que afectan a su evolución, a lo largo de una década, pero también a la situación actual, tanto para el Catastro de Rústica como para el de Urbana, en función de la concentración de la 
propiedad y de la riqueza catastral. Para el Catastro de Rústica hemos tenido en cuenta la totalidad de los municipios de España, es decir 8.110, incluidos los 535 de las cuatro provincias forales, información solicitada personalmente a dichas administraciones; respecto al Catastro de Urbana se hace referencia a las capitales de provincia y municipios con más de cincuenta mil habitantes (Pillet, 2008 a y b). La información aportada por la Dirección General desde 1990, ha variado a partir de 2006 al presentar variaciones en sus variables, que no son del caso ahora, pues hemos utilizado las que hacen referencia al tránsito de un siglo a otro (1995-2005). El aspecto más importante a tener en cuenta es el valor catastral, pues se ha convertido en el centro del análisis por ser una de las figuras jurídicas de mayor impacto social para valorar los bienes inmuebles según Varona (2001).

Analizada la evolución de las fuentes y su tratamiento para el estudio de la propiedad rústica y urbana, hicimos un análisis comparativo sobre la situación general de las comunidades autónomas actualmente (Pillet, 2007), de él y de lo estudiado a nivel nacional se desprede que Castilla-La Mancha ha destacado en la concentración de la propiedad rústica, mientras que por el contrario su situación es muy negativa en la riqueza catastral urbana, tal y como vamos a estudiar. Mientras las publicaciones citadas sobre propiedad serán recogidas en el texto, las que hacen referencia a su riqueza territorial las podemos encontrar, gran parte de ellas, en el reciente balance realizado sobre la investigación geográfica en la universidad regional por Cañizares (2009).

\section{LA EVOLUCIÓN DE LA PROPIEDAD RÚSTICA: LA PRESENCIA DE GRANDES FINCAS}

Los primeros estudios sobre la propiedad rústica de Castilla-La Mancha, en su conjunto, los realizaron Antonio López Ontiveros (1978) para "Castilla la Nueva y Albacete", y en segundo lugar, una década después, Rafael Mata en el encuentro sobre El espacio rural de Castilla-La Mancha $(1988)^{1}$. Desde entonces esta temática no ha vuelto a tratarse de forma general, por este motivo nos parece acertado hacer un análisis de la evolución seguida desde los orígenes hasta la actualidad, pues existen suficientes obras, así como nuevos elementos que podemos añadir, como complemento a la introducción de las fuentes catastrales, a las que hacíamos referencia en 2007.

1.Encuentro coordinado por F. Pillet en la que impartieron las conferencias de apertura y clausura, los profesores Antonio López Gómez y Joaquín Bosque, respectivamente; y las ponencias sobre Castilla-La Mancha: Mercedes Molina (la población), Miguel Ángel Troitiño (el poblamiento rural), Manuel Valenzuela (la ordenación de los espacios rurales), Fernando Arroyo (la innovación agraria) y Rafael Mata (la propiedad agraria). 


\subsection{LA EVOLUCIÓN DE LA PROPIEDAD HASTA LOS AÑOS OCHENTA DEL SIGLO XX}

La repoblación y la ocupación de las tierras en época medieval originaron una situación diferente a la ocurrida en Andalucía, debido a la facilidad para acceder a la propiedad de la tierra en la edad moderna, por el escaso número de habitantes desde el siglo XVI (López-Salazar, 1986: 278-280). Todo ello dio como resultado una abrumadora porción de superficie agraria (García y Gómez, 2010: 107), concentrada en manos de las Órdenes Militares, de la Iglesia, de la nobleza local, así como de grupos pertenecientes a caballeros, hidalgos y grandes labradores (Donézar, 1984), en su mayoría hidalguías o de bajo nivel a mediados del siglo XVIII.

La reforma liberal a esta situación vino de la mano de la desamortización, en estas tierras Quirós (1964) escribió la primera obra con el fin de medir la intensidad del fenómeno en un territorio concreto: Valle de Alcudia y Campo de Calatrava, recogida posteriormente en facsímil (Quirós, 1992), obras posteriores fueron analizando la situación en las provincias, bibliografía muy conocida que no podremos recoger. Las consecuencias de la misma se pueden seguir en primer lugar, en los listados de la Riqueza territorial, donde se observa la desaparición de las propiedades eclesiásticas, la pervivencia de títulos de rancio abolengo con raíces en el Antiguo Régimen, junto a la naciente burguesía que se benefició con la desamortización (Mata, 1988: 178-180). Actualmente contamos con nuevos análisis que permiten un conocimiento más general de la región. Los mayores remates se dieron en las provincias de Toledo y Ciudad Real, pues su volumen se rigió más por la extensión de las propiedades del clero y de los propios-baldíos (Feijoo, 1990: 127-129). Con datos ya más recientes, se puede afirmar que la superficie vendida afectó en la región a un total de 1,7 millones de hectáreas, siendo la provincia más afectada Ciudad Real (757.211 has), seguida de Toledo (329.335), y a continuación: Albacete, Guadalajara y Cuenca. La nobleza provinciana, la burguesía madrileña y regional controlaban el $70 \%$ de la cuota de los mayores contribuyentes en 1875, protagonismo que iría en aumento (Valle, 2010: 156-159).

En los años treinta del pasado siglo, primero la "memoria" elaborada por la Dirección General de Propiedades y Contribución Territorial (1930) y luego el Registro de la Propiedad Expropiable dejaron clara la situación de las tres provincias más significativas: Ciudad Real se encontraba a la cabeza de España con el mayor número de fincas superiores a mil hectáreas (García Badell, 1946:179), su mayor propietaria, perteneciente a la nobleza reunía unas cincuenta mil hectáreas, al tiempo que los bienes expropiados a la grandeza de España se centraron en dos nobles de la provincia de Ciudad Real y tres de Toledo (Ladrón de Guevara, 2010: 249-251); por su parte en Albacete, Espejo confirma que las tierras expropiables suponen una cuarta parte del total provincial, la mayor parte absentistas que residen, sobre todo en Madrid y Valencia, pertenecientes todos ellos a la nobleza (Espejo, 1990 y 1991).

Con la contrarreforma llevada a cabo tras el conflicto bélico, López Ontiveros (1978) presenta un balance evolutivo para finales de los setenta, donde indica la situación de las diferencias provinciales: Ciudad Real y Toledo ofrecen grandes fincas en el montuoso occidente, continuación del latifundio extremeño; Albacete las localiza- 
ba no sólo en montaña sino también en llanura; mientras que Guadalajara y Cuenca se caracterizan por un minifundismo acentuado; un dato de interés es la afirmación de que la gran propiedad ha sido actora principal de la expansión del regadío "se ha vencido, pues, por la gran propiedad, la alergia tradicional hacia el regadío", especialmente en la gran comarca geográfica de La Mancha, como se ha podido comprobar en Ciudad Real (Pillet, 1989a: 141-142), y en Albacete (Espejo: 1989: 174). La concentración parcelaria afectó con mayor intensidad a Guadalajara y Cuenca, menos a Albacete y Toledo, y de manera imperceptible a Ciudad Real, su expansión se debió a que fueron los grandes propietarios los más interesados en que se llevase a cabo ya que una parte de sus tierras las tenían arrendadas (Vázquez, 1988).

La visión general más completa nos lo proporciona la información de los últimos años del Censo de Exploraciones sujetas a Cuota Proporcional (1965-1978), en el que llama la atención que las explotaciones más valoradas (2.657) coincidan con las provincias que presentan mayor número de grandes fincas (Albacete, Ciudad Real y Toledo), pero cuando hemos descendido a las 80 que sobresalen nos han aparecido en primer lugar las provincias de Guadalajara y Cuenca. Esta situación no se corresponde ni con el pasado ni con la actualidad catastral de estas provincias, también es cierto que para Cuenca y concretamente para su Ayuntamiento se ofrece una valoración excesiva que rompe con la información general. Con independencia de lo dicho, los cuadernillos provinciales se convirtieron en el mejor catálogo para el conocimiento de las grandes propiedades, el aspecto más negativo fue que la reforma de 1978 suprimiría dicho Censo de Explotaciones (Pillet, 2007: 216-219).

\subsection{LA DIVERSIDAD REGIONAL A TRAVÉS DEL CATASTRO DE RÚSTICA: COMARCAS SERRANAS Y DE LLANURA}

Pasaremos ahora a contemplar la situación de una serie de comarcas representativas, comenzando en primer lugar por los principales ejemplos de grandes fincas rústicas en comarcas serranas, en Los Montes y en Sierra Morena-Valle de Alcudia, ambas en la provincia de Ciudad Real, relacionando el Catastro con el Registro de la Propiedad. Y en segundo lugar, las situadas en la llanura, especialmente la gran comarca de La Mancha, a lo largo de cuatro provincias, todas excepto Guadalajara, a la que acompañaremos distintos trabajos como consecuencia de tesis doctorales de geografía agraria que tuvieron su punto de partida en el Catastro de Ensenada y que concluyen con el Catastro Parcelario en los años setenta u ochenta del siglo pasado.

Coincidiendo con la ponencia sobre el marco jurídico de la propiedad rústica en el V Coloquio de Geografía Agraria de Santiago de Compostela (1989) pudimos informar sobre la situación de la comarca agraria de los Montes Norte o Montes de Ciudad Real por la importancia de las grandes fincas allí existentes (Pillet, 1989 b), esta situación podía completarse con otros ejemplos como los situados en la comarca agraria de Los Pastos (Pillet, 1988 b y 1991) pues ambas se encuentran enclavadas, respectivamente, en las comarcas geográficas de Los Montes de Toledo y Ciudad Real y en Sierra Morena-Valle de Alcudia. En ellas coincide la gran propiedad con importantes cotos de caza mayor (ciervo y jabalí) tal y como estudió Martínez Garrido (1991 y 2000) y posteriormente esta misma autora con Torrija (2009: 1174-75). 
En un listado proporcionado por la Delegación de Hacienda de Ciudad Real (diciembre-1994) con 234 fincas de más de mil hectáreas hemos comprobado, para esta ocasión, que la mayor parte de las que superan las 5.000 hectáreas se encuentran en las comarcas citadas.

En la comarca de Los Montes de Ciudad Real o Montes Norte, junto a Cabañeros, que hemos analizado siguiendo el Registro de la Propiedad y que es el resultado de las fincas que pasaron con la desamortización a manos privadas, sus 26.432 has de dehesa han dado lugar al Parque Nacional con 40.000 has (Pillet, 2008 c: 139-142). Otro ejemplo, también estudiado por diversos autores es la situación singular de los Estados del Duque o Montes y Terrenos del Estado de Malagón que se extienden por cinco municipios de la provincia de Ciudad Real (Los Cortijos, Fuente el Freno, Malagón, Porzuna y El Robledo) sumando un total de 18.617 hectáreas, considerados como una verdadera pieza de arqueología jurídica (Sanz, 1976), con derechos históricos sin resolver (Mangas, 1988), a la vez que tiene sumidos a sus vecinos en la inseguridad jurídica según Santos (2010), al que dirigimos su tesis doctoral. Junto a estos dos casos, en esta misma comarca, se encuentran las fincas de personalidades de la nobleza y las finanzas; en Puebla de Don Rodrigo, existe una gran finca del Ayuntamiento (16.573 has) y otra de la Junta de Comunidades de Castilla-La Mancha (6.102 has); más una de propiedad privada en Piedrabuena (7.476 has).

La comarca geográfica Sierra Morena-Valle de Alcudia, engloba dos comarcas agrarias, en la parte occidental (Los Montes Sur) y en la parte oriental (Los Pastos). En esta última destacan la finca La Garganta, de 11.000 has, adquirida en propiedad por la Sociedad Minero Metalúrgica de Peñarroya, y situada en los municipios de Almodóvar del Campo y Brazatortas; perteneció al proceso desamortizador, siendo vendida en 1971 a una empresa filial. También existen en Viso del Marqués, una perteneciente a su Ayuntamiento (12.318 has), y otra a una sociedad (5.249 has); así como en Fuencaliente una del Ayuntamiento (10.089 has), y en Mestanza una de propiedad privada (5.792 has). En la parte occidental (Montes Sur) destaca la dehesa o Encomienda de Castilseras, con una superficie de 8.435 has localizada en los municipios de Almadén, Almadenejos y Alamillo, en 1905 se inscribió a nombre del Estado y en 1954 pasaría bajo el control del Consejo de Administración de Minas de Almadén y Arrayanes, organismo dependiente del Ministerio de Hacienda; en este municipio destaca una finca privada (5.654 has). Con esta relación se puede comprobar la importancia de las grandes fincas en las comarcas serranas, caracterizadas por la gran concentración de la propiedad rústica.

La gran llanura de La Mancha se extiende por más de noventa municipios a lo largo de cuatro provincias, comarca sobre la que hemos analizado su evolución catastral en tres momentos distintos: 1978, 1988 y 1996 (Pillet, 2001: 63-80), y tras comprobar el paso del secano al regadío, se llega a la conclusión, que si relacionamos los municipios de La Mancha con mayor riqueza catastral con los que ofrecen mayor concentración de la propiedad, se puede ver cómo la mayor concentración de la propiedad se da en el extremo suroriental (acuífero 18) siendo La Herrera, Albacete y Barrax los municipios más destacados, mientras que las de mayor rique- 
za aparecen en el centro y en el extremo suroccidental (acuífero 23) tal es el caso de Pedro Muñoz, Socuéllamos y Villarta de San Juan los más relevantes.

Tres tesis doctorales de geografía agraria sobre comarcas de llanura, que parten del Catastro de Ensenada para concluir en el Catastro Parcelario de los años ochenta del siglo pasado, analizaron, entre otros aspectos, la influencia de las mayores propiedades: la Sagra (Rodríguez, 1984), la Mancha conquense (Gesteiro, 1990 y 1996) y el Campo de Calatrava (Rodríguez, 2000); y en un contexto más amplio también se estudiaron la Campiña de Guadalajara (Gómez Mendoza, 1977) y la Mancha de Albacete (Romero, 1983) En todas ellas el protagonismo de la gran propiedad ha sido importante, excepto en la Campiña del Henares.

\section{LA ACTUAL VALORACIÓN CATASTRAL RÚSTICA: UNA SITUACIÓN DUAL}

El actual Catastro Inmobiliario nos sigue aportando la información tradicional a partir de los libros de células de propiedad, que ya utilizamos con el Catastro Parcelario (1985) y que ahora Francisco Feo ha estado trabajando desde una perspectiva de género en tres provincias de la región (Toledo, Guadalajara y Cuenca). La provincia que destaca por el mayor porcentaje de propietarios y controla mayor superficie es Toledo, mientras que en número de propietarias y mayor superficie registrada aparecen Guadalajara y Cuenca (Feo, 2001: 213). Mirando las restantes provincias españolas que ha estudiado, se podría decir, que mayoritariamente donde predomina la gran propiedad destacan los propietarios, mientras las propietarias se hacen más visibles en la mediana y pequeña propiedad.

La Dirección General del Catastro viene publicando estadísticas catastrales desde 1990, información que nos ha permitido poder comparar Castilla-La Mancha con el resto de regiones de España, aspecto que no era posible antes, al tiempo que se puede descender por las restantes escalas: provincias y municipios. Con los datos aportados venimos relacionando el valor catastral con el número de titulares para conocer la concentración de la propiedad; al mismo tiempo que al poner en contacto el valor catastral con la superficie podemos hablar de la riqueza catastral. Las estadísticas nos han permitido, también, comprobar que no existen diferencias importantes, en relación con la fecha de renovación catastral.

\subsection{LA CONCENTRACIÓN DE LA PROPIEDAD RÚSTICA}

Aunque es Andalucía y la provincia de Sevilla las que ocupan el primer lugar de España en concentración de la propiedad rústica, en páginas anteriores hemos comprobado que Castilla-La Mancha tiene una presencia destacada, por encima de la media nacional; como así lo demuestran, también, las importantes ayudas o subvenciones que la PAC dirige a grandes propietarios que radican en estas tierras sus fincas rústicas, muchas de ellas situadas en las comarcas que hemos comentado. 
Aunque el mayor crecimiento durante una década (1995-2005) lo ha experimentado la provincia de Albacete, seguida de Ciudad Real, en el último año de estudio (2005) el primer lugar en concentración lo sigue ostentando la provincia de Ciudad Real, seguida de Albacete y Toledo. Los municipios que sobresalen en concentración en dichas provincias por superar los 25 de valor catastral $/ \mathrm{n}^{\circ}$ de titulares son: en Ciudad Real, Llanos del Caudillo, poblado de colonización que se segregó de Manzanares, y Los Pozuelos de Calatrava; en Albacete, La Herrera; y en Toledo, Barcience y el mismo Toledo. Barcience, aunque aparece destacado en concentración, ofrece una situación irregular, pues sólo cuenta con nueve titulares catastrales, situación muy poco común, y además no renovó su catastro.

Para poder adentrarnos en la realidad de Castilla-La Mancha descenderemos al nivel supramunicipal, utilizando la comarcalización geográfica que hicimos años antes con Panadero (Panadero y Pillet, 1999), y que ahora se ha presentado el mapa resultante con sus correspondientes municipios (Pillet, 2010b), organizadas en tres bloques: Comarcas de Llanura, de Transición o piedemonte y de Sierra (anexo1), comarcalización que será recogida en el Atlas de los paisajes de Castilla-La Mancha que publicará la Consejería de Ordenación del Territorio y Vivienda de la Junta de Comunidades de la región; y sobre laque recientemente ha estudiado su población Monteagudo (2010).

Si la media de concentración de la propiedad en la región es de 3,4 V/T (anexo 2) tan sólo el conjunto de comarcas de llanura la superan $(5,4 \mathrm{~V} / \mathrm{T})$, quedado por debajo las de transición o piedemonte $(3,6 \mathrm{~V} / \mathrm{T})$ y las de sierra $(2,5 \mathrm{~V} / \mathrm{T})$. De donde se desprende, que no sólo nos estamos refiriendo a zonas favorables al desarrollo agrario, sino que además presentan la propiedad muy concentrada.

La comarca de llanura (figura 1) que alcanza el lugar más destacado de la región es la Vega de Toledo $(8,9 \mathrm{~V} / \mathrm{T})$ en ella aparecen cuatro municipios que merecen ser citados: Toledo, Malpica del Tajo, Mocejón y Albarreal del Tajo. Las restantes comarcas son, primero la Tierra de Torrijos $(7,7 \mathrm{~V} / \mathrm{T})$, perteneciente también a la llanura toledana, lo que viene a incidir en una provincia claramente caracterizada históricamente por la gran propiedad, encontrándose en ella el municipio de Barcience, del que ya hemos destacado su elevada sobrevaloración, al que acompaña Rielves. La tercera comarca, de transición o piedemonte, en la provincia de Ciudad Real, y caracterizada por la gran propiedad, es el Campo de Calatrava $(6,3 \mathrm{~V} / \mathrm{T})$ con los municipios de Los Pozuelos de Calatrava, Ciudad Real y Corral de Calatrava. Si hasta ahora todas las comarcas están situadas en una misma provincia, tanto en Toledo como en Ciudad Real, la comarca que aparece a continuación es la gran llanura o subregión de La Mancha que se extiende por cuatro provincias, ejemplo claro de comarca de llanura con una concentración de 5,4 V/T, donde citaremos a los municipios de La Herrera en Albacete y Llanos del Caudillo en Ciudad Real. 
Figura 1. La concentración de la propiedad rústica (V/T) en las comarcas geográficas de Castilla-La Mancha

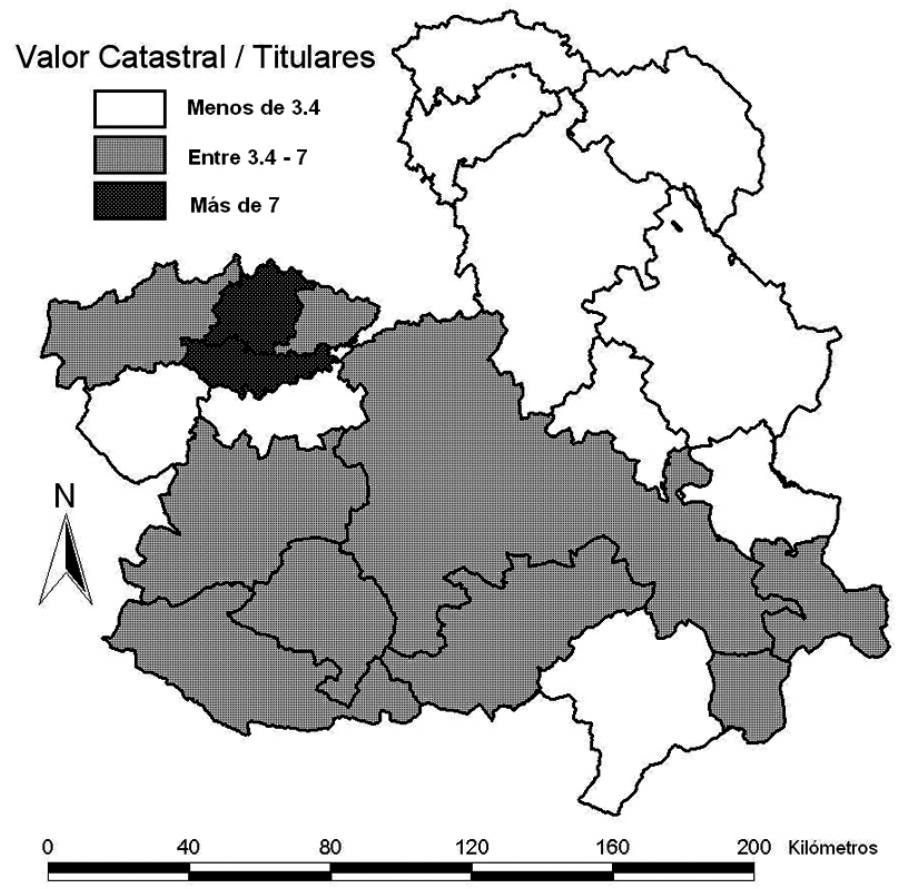

Fuente: Dirección General del Catastro, 2005 (Elaboración propia)

Las comarcas que vienen a continuación: La Sagra $(4,6 \mathrm{~V} / \mathrm{T})$, el Corredor de Almansa (4,5), Campos de Hellín (4,4), Sierra Morena y Valle de Alcudia $(4,1)$ y Los Montes de Toledo y Ciudad Real $(4,1)$, se encuentran tanto en llanura, transición como en sierra, respectivamente y fueron citadas algunas de ellas en el análisis evolutivo que hicimos. Las restantes comarcas quedan por debajo de la media de concentración rústica y se localizan mayoritariamente en el noreste y este de la región, una en el sur y dos en el oeste. La que presenta menor concentración de la propiedad rústica es la Sierra de Guadalajara $(0,8)$, todas ellas ejemplos claros de zonas caracterizadas por las medianas y pequeñas propiedades, lo que marca una clara dualidad como se observa en la figura que estamos analizando.

\subsection{LA RIQUEZA CATASTRAL RÚSTICA}

La Comunidad Valenciana y especialmente la provincia de Valencia, seguida de Almería son las que representan la mayor riqueza catastral de España. La evolución de dicha riqueza (1995-2005) sitúa a Castilla-La Mancha ligeramente por debajo (0,4 V/S) de la media nacional $(0,5 \mathrm{~V} / \mathrm{S})$ según la información previamente publicada. Pasando ahora a contemplar la totalidad de los municipios de Castilla-La Mancha y sus correspondientes provincias, se comprueba que las tres que presentan mayor riqueza son, como ya ocurría con la concentración, Albacete, Ciudad Real y Toledo. La diferencia entre ellas nos la marcará la importancia de los municipios que ofrecen una riqueza 
superior a 1,5 V/S: en primer lugar aparece la provincia de Toledo, con Miguel Esteban, Añover del Tajo y Villaseca de la Sagra; en segundo lugar, Ciudad Real con Llanos del Caudillo y Pedro Muñoz; y en tercer lugar, la provincia de Albacete, con La Herrera.

Como hacíamos con la concentración, analizaremos el nivel supramunicipal, es decir las comarcas geográficas. Si la media de riqueza rústica de Castilla-La Mancha registra un 0,4 de valor catastral respecto a la superficie $(\mathrm{V} / \mathrm{S})$, a la cabeza se sitúan las comarcas de llanura con 0,6 de media, y le siguen las de transición o piedemonte $(0,4)$, quedando a gran distancia las de sierra $(0,1)$, resultado lógico pues es la llanura la sede de la riqueza agraria.

Las tres comarcas más valoradas se localizan en llanura (figura 2), ocupando la parte central de la región, en dirección noroeste-sureste, nos referiremos a la Vega de Toledo $(0,8 \mathrm{~V} / \mathrm{S})$, La Sagra $(0,7 \mathrm{~V} / \mathrm{S})$ y La Mancha $(0,7 \mathrm{~V} / \mathrm{S})$, las dos primeras se corresponden con los regadíos de superficie de la cuenca del Tajo, y la tercera con los acuíferos de la cuenca del Guadiana, estudiada esta última en la tesis que hemos dirigido (Ruiz Pulpón, 2007). En La Vega de Toledo sobresalen los municipios de Mocejón, Montearagón y Cebolla; en La Sagra, citaremos a Añover del Tajo, Villaseca de la Sagra y Recas; y ya por último, en La Mancha, Llanos del Caudillo, Pedro Muñoz y La Herrera. En situación opuesta aparecen, entre otras, la Sierra de Guadalajara, Molina de Aragón y la Serranía de Cuenca, con escasa riqueza catastral, tal y como se observa en la figura.

Figura 2. La riqueza catastral rústica (V/S) en las comarcas geográficas de Castilla-La Mancha

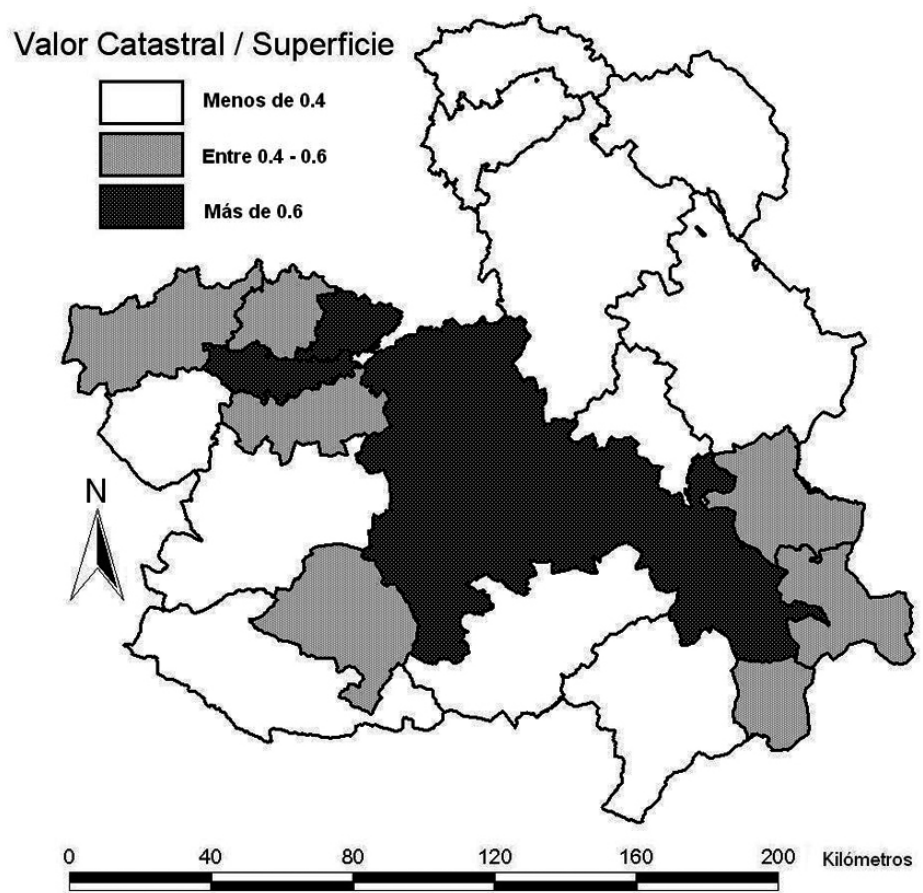

Fuente: Dirección General del Catastro, 2005 (Elaboración propia) 
El "problema de la tierra" cobra un lugar importante en tiempos de crisis económica cuando otros sectores no son capaces de generar empleo, aunque la situación en Castilla-La Mancha no es comparable con la problemática andaluza (Martín, 2008). En nuestra región tanto históricamente como ahora se da una situación dual, debido a sus grandes contrastes, pues frente a tres provincias con predominio de grandes fincas, aunque con claras diferencias internas (secano en sierra frente a regadío en llanura) aparecen zonas de dominio de pequeñas y medianas propiedades, con baja concentración y escasa riqueza.

El Catastro de Rústica nos presenta para la región que tres provincias han destacado tradicionalmente por el número y la dimensión de sus fincas: Ciudad Real, Toledo y a continuación Albacete. Hemos comprobado que en las comarcas serranas donde se encuentran las fincas donde se supera las mil o las cinco mil hectáreas como eran por ejemplo Los Montes de Ciudad Real o Montes Nortes, así como Sierra Morena-Valle de Alcudia, a la hora de analizar actualmente la concentración catastral, no destacan; pues las comarcas que ocupan el primer lugar en concentración de la propiedad y de la riqueza catastral se encuentran en la llanura, nos referimos para el primer caso a la Vega de Toledo y a la Tierra de Torrijos, ambas en la provincia de Toledo; de la misma forma que las que presentan mayor riqueza catastral son $\mathrm{La}$ Vega de Toledo y La Sagra, también en la provincia de Toledo, a ellas se une La Mancha que se extiende por cuatro provincias. Los dos municipios, de pequeño tamaño, que mejor simbolizan la mayor concentración y riqueza se encuentran en $\mathrm{La}$ Mancha: Llanos del Caudillo (Ciudad Real) y La Herrera (Albacete).

\section{LA EVOLUCIÓN DE LA PROPIEDAD Y LA VALORACIÓN CATASTRAL URBANA: UNA RIQUEZA CATASTRAL MUY ESCASA}

Los estudios sobre propiedad urbana han sido mucho más reducidos que los realizados sobre la propiedad rústica, de hecho en Castilla-La Mancha solo existen tres obras, tesis doctorales que analizaron tres capitales de provincia explicando la estructura de la propiedad y el valor del suelo desde el Catastro de Ensenada hasta la fecha de publicación, nos referimos a Guadalajara (García Ballesteros, 1978), Cuenca (Troitiño, 1984) y Ciudad Real (Pillet, 1984). Posteriormente en un artículo se estudiaba la misma evolución de la propiedad para un municipio que fue capital de provincia (1750-61) y que posteriormente lo volvió a intentar nuevamente como ocurrió en otras provincias en 1837 (Pillet, 1987) nos referimos a Almagro (Pillet, 1988). Y por último, existen dos estudios sobre la desamortización, el que relaciona Guadalajara con Plasencia (García Ballesteros y Redondo, 1983) y un estudio histórico sobre las consecuencias de la desamortización urbana en Toledo (Cerro, 1988). Desde entonces parece como si no haya interesado el estudio de la propiedad urbana a partir del Catastro, como comentábamos en la introducción.

De los cuatro momentos históricos que se reflejan en las obras citadas: el Catastro de Ensenada de mediados del siglo XVIII, el proceso de desamortización y sus consecuencias, la estructura de la propiedad hasta los años treinta del siglo XX, y la propiedad urbana según el Catastro Parcelario, desde los años setenta hasta finales de los ochenta, podemos constatar que la evolución nos muestra que primero fue 
la Iglesia y la nobleza, luego una burguesía local y por último una burguesía inmobiliaria y financiera la que ha regido el control de la propiedad urbana. Se puede afirmar que conforme ha ido avanzando el tiempo un menor número de propietarios registran un mayor porcentaje de riqueza urbana.

El Catastro Inmobiliario actual de Urbana nos presenta en primer lugar, tanto en concentración de la propiedad como en riqueza a la Comunidad de Madrid. La década que venimos analizando (1995-2005) ofrece en Castilla-La Mancha una concentración de la propiedad urbana $(0,04 \mathrm{~V} / \mathrm{T})$ algo inferior a la media nacional $(0,06) \mathrm{y}$ por el contrario una riqueza $(0,04 \mathrm{~V} / \mathrm{S})$ muy por debajo de la nacional $(0,12)$, con consecuencias llamativas como veremos a continuación.

\subsection{LA CONCENTRACIÓN DE LA PROPIEDAD URBANA}

La concentración de la propiedad urbana actual en sus correspondientes provincias muestra una situación similar en todas, excepto en Cuenca que es peor. El número de municipios con más de $80 \mathrm{~V} / \mathrm{T}$ nos ayuda a comprender las diferencias entre las provincias, existen dos que se distancian del resto, nos referimos a Toledo y Guadalajara, es decir las más próximas a Madrid, ambas presentan seis municipios destacados. En el caso de Toledo, citaremos en primer lugar los núcleos urbanos más importantes, el mismo Toledo, junto a los municipios rurales de Cobisa, Esquivias, Méntrida, Pepino y Yeles. En Guadalajara, a Azuqueca de Henares le acompañan Alovera, Chiloeches, Junquera de Henares, Pozo de Guadalajara y Trillo. En la provincia de Ciudad Real, Valdepeñas. En Albacete, Villalgordo del Júcar. Y ninguno en Cuenca.

Para un análisis más detallado nos parece oportuno centrarnos en las comarcas geográficas. La única que supera la media regional son las comarcas de llanura con un $0,06 \mathrm{de} \mathrm{V} / \mathrm{T}$ (anexo 2); quedando en situación similar, las de transición o piedemonte con 0,04 y por debajo las de sierra $(0,03)$.

A la hora de relacionar el valor catastral con los titulares catastrales, se ha pasado el valor catastral de miles de euros a millones de euros. Ocupando un lugar muy destacado en concentración de la propiedad (figura 3) aparece una comarca de llanura, nos referimos a La Sagra (0,08 V/T), con Yeles y Esquivias como municipios más importantes, dicha comarca se encuentra lindando con la Comunidad de Madrid, como así ocurre también con las cuatro primeras comarcas que citaremos a continuación.

Para las restantes comarcas $(0,06$ a 0,04$)$, destacaremos del grupo las que presentan un 0,06 V/T, todas ellas de llanura excepto La Sisla: la Vega de Toledo con Toledo y Argés; La Mancha con Valdepeñas y Miguel Esteban; la Tierra de Torrijos con Méntrida; y La Sisla con Cobisa. A continuación con 0,05: Talavera de la Reina y Oropesa y Campo de Calatrava; con 0,04 aparecen la Campiña del Henares, La Manchuela, el Corredor de Almansa, el Campo de Hellín, el Campo de Montiel, Sierra Morena y Valle de Alcudia, más Los Montes de Toledo y Ciudad Real. Nuevamente, las comarcas siguientes, valoradas por debajo de la media regional vuelven a situarse en los mismos lugares donde la valoración de rústica era inferior, tal y como se observa en la figura 3 , es decir, en las zonas montañosas y de piedemonte. 
Figura 3. La concentración de la propiedad urbana (V/T) en las comarcas geográficas de Castilla-La Mancha

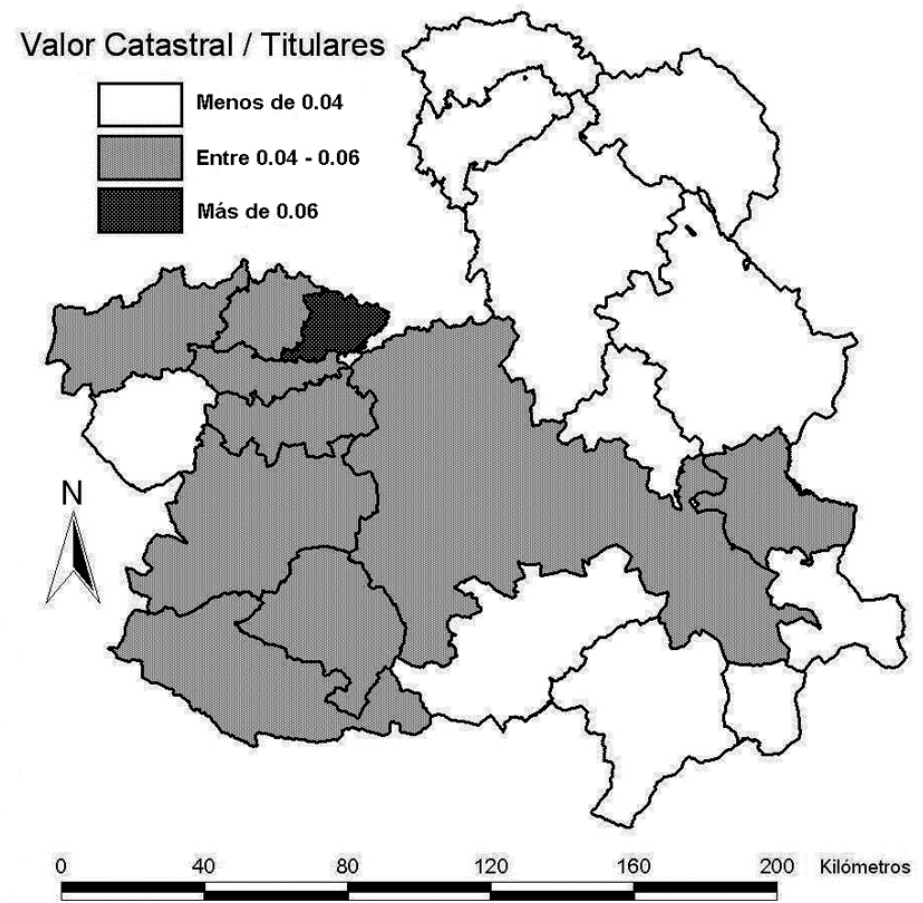

Fuente: Dirección General del Catastro, 2005 (Elaboración propia)

\subsection{LA RIQUEZA CATASTRAL URBANA}

Aunque la provincia más valorada es Albacete $(0,07 \mathrm{~V} / \mathrm{S})$, le siguen muy de cerca Ciudad Real $(0,06)$, Cuenca $(0,05)$, Guadalajara $(0,04)$ y Toledo $(0,03)$, la diferencia es muy escasa de unas a otras (Pillet, 2007: 223), todas ellas se encuentran por debajo de la media nacional $(0,12)$. Peor situación ofrecen algunas capitales y municipios de más de cincuenta mil habitantes, si nos fijamos en las capitales, el último lugar de España lo ocupa Ciudad Real $(0,10)$, seguido de Toledo $(0,11)$ y Albacete $(0,12)$; peor situación presenta Puertollano $(0,08)$. Un lugar más destacado lo ofrece Guadalajara $(0,19)$ y Talavera de la Reina $(0,15)$, y en situación intermedia aparece Cuenca $(0,13)$. De esta forma las tres capitales de España peor valoradas son Ciudad Real, Toledo y Ávila con 0,11 V/S (Pillet, 2008 b: 6 y 16).

Si nos detenemos en las cinco capitales y en los dos municipios de más de cincuenta mil habitantes de la región comprobamos que sus catastros han sido renovados entre 1996 y 1997, sólo en Ciudad Real, que está en peor situación, la renovación fue posterior (2000), por lo tanto la razón no la podemos encontrar en la fecha de renovación catastral ¿Cómo podemos explicar el desafuero de la situación en las capitales, en Puertollano y muy especial en Ciudad Real que ocupa el último lugar de España?. 
Para dar respuesta a esta pregunta, haremos referencia en premier lugar, a Barcelona, que ocupa el primer lugar de España en riqueza catastral y que nuevamente ha vuelto a ser la ciudad de los prodigios. Siempre recordaremos de ella, a pesar de los problemas que señaló Capel (2007) que puso en funcionamiento el primer plan estratégico de ciudades con motivo de los juegos olímpicos.

De Ciudad Real, que ocupa el último lugar, tendremos que recordar que desde el Plan General de Ordenación Urbana de 1963 (Pillet, 1984: 438), hasta el reciente avance del Plan de Ordenación Municipal, se ofrecen proyecciones demográficas falsas o exageradas para justificar miles de nuevas viviendas, entonces esto podría ser creíble, pero ahora la planificación urbanística ya no puede seguir por ese camino, tras la crisis. Algunos señalan, para defender estas proyecciones, que a Ciudad Real le podría pasar lo que le ocurrió a Albacete, que absorbió gran parte de la población provincial, pero son situaciones diferentes, pues, como a continuación veremos, la provincia de Ciudad Real presenta centros urbanos importantes y es muy difícil que pueda acontecer lo mismo. En Ciudad Real no solo está en avance el Plan urbanístico, sino también desde 2005 el Plan Estratégico Ciudad Real 2015, que aparece y desaparece, aunque ahora debe estar dormido, cuando es más necesario, pues ambos deben andar conjuntamente, uno proyectando de cara a doce años y el otro para unas décadas, respectivamente.

Ante la situación que presentan las principales ciudades de la región, es necesario un estudio en profundidad, a las ya citadas anteriormente, que analizaron la evolución de la propiedad, añadiríamos ahora distintas tesis y monografías existentes. Comenzando por Albacete, citaríamos en primer lugar a Panadero (1976), y posteriormente a García Martínez (1995) y Losada (2000); en Ciudad Real, Pillet (1995 y 2005) y la tesis que hemos dirigido sobre las últimas décadas, actualmente en prensa (Rodríguez Doménech); en Cuenca, Troitiño (1998); en Guadalajara, García Roldán (1992); en Puertollano, Cañizares (1998); en Talavera de la Reina, Mejías (2008) y en Toledo, Zárate (1984, 2000, 2007 y 2010) y Campos (1996 y 2010). Para terminar, haremos referencia a dos reflexiones generales, sobre el urbanismo regional (Coronado, Pillet y Ureña, 2004), y sobre sus ciudades (García Martínez y Vázquez, 2007).

Las cinco capitales, más Puertollano y Talavera de la Reina, junto a Alcázar de San Juan-Tomelloso, Illescas y Valdepeñas las hemos incluido en el policentrismo de Castilla-La Mancha con sus correspondientes áreas funcionales urbanas integradas por un centro y distintos subcentros (Pillet, et al 2010), teniendo como soporte la tesis doctoral sobre transporte y territorio que hemos dirigido (Martínez, 2010). La situación de los principales municipios es distinta entre ellos: Toledo es la capital de la comunidad autónoma y Albacete es el único que supera los ciento cincuenta mil habitantes. Cuenca y Toledo son ciudades Patrimonio de la Humanidad. Ciudad Real y Toledo unen en su espacio provincial distintos núcleos del policentrismo y presentan un porcentaje más elevado de población vinculada (22\%), por el contrario, Puertollano, Talavera de la Reina y Albacete son la que atraen menor porcentaje de población vinculada. Guadalajara y Talavera de la Reina son las que presentan mayor riqueza catastral urbana, la primera vive en estrecha conexión con Madrid. 
Para terminar, dejaremos estos municipios urbanos importantes para centrarnos en las comarcas, advirtiendo en primer lugar que no sobresale ninguna, lo que demuestra la escasa riqueza urbana (figura 4). En esta situación general, en llanura, destaca La Mancha, con Valdepeñas y Miguel Esteban; y en transición o piedemonte, La Manchuela, con Villamalea. De las restantes, citaremos las dos que presentan los municipios de más de cincuenta mil habitantes más valorados catastralmente, en la Vega de Toledo, Talavera de la Reina y en la Campiña del Henares, Guadalajara. Es decir, estos dos municipios más Valdepeñas, son los municipios urbanos más valorados en riqueza catastral.

El Catastro de Urbana presenta, con mayor concentración de la propiedad a las provincias más próximas a Madrid: Toledo y Guadalajara, así como una comarca de la primera provincia que linda con dicha comunidad, nos referimos a La Sagra, aunque su situación en riqueza catastral es muy negativa. La primera provincia en riqueza catastral es Albacete con su capital; así como la comarca más valorada es La Mancha, donde Valdepeñas sobresale en concentración y riqueza. Y por último, es necesario señalar la situación negativa que presentan Ciudad Real, Toledo y especialmente Puertollano.

Figura 4. La riqueza catastral urbana (V/S) en las comarcas geográficas de Castilla-La Mancha

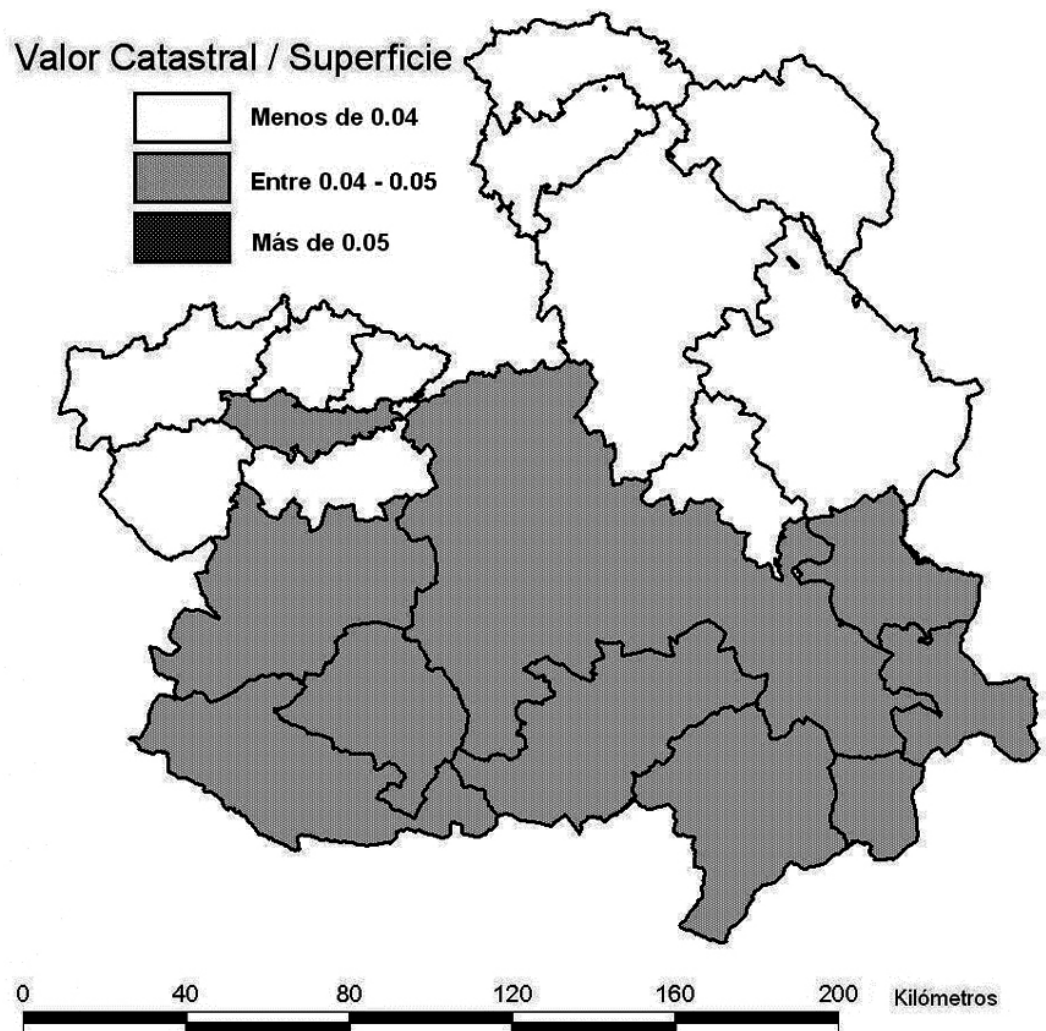

Fuente: Dirección General del Catastro, 2005 (Elaboración propia) 


\section{CONCLUSIÓN}

El estudio evolutivo de la propiedad rústica y urbana desde mediados del siglo XVIII hasta la actualidad nos permite afirmar que, con datos del Catastro de Rústica, no existe coincidencia entre la localización de las grandes fincas rústicas de Castilla-La Mancha, en función de su tamaño, con las comarcas donde actualmente se da la mayor concentración catastral de la propiedad rústica, en función del valor catastral, siendo la región superior a la media nacional. Respecto a la riqueza catastral, tanto de rústica como de urbana, la situación de la región es muy negativa, especialmente en esta última pues dos de sus capitales ocupan los últimos lugares de España, nos referimos a Ciudad Real y Toledo, coincidiendo en esta circunstancia con otra capital castellana: Ávila.

Cuatro comarcas destacan sobre el resto: La Vega de Toledo en concentración de la propiedad y de la riqueza rústica; La Sagra, en propiedad urbana y riqueza rústica, La Mancha, en riqueza rústica, en concentración urbana y escasamente en riqueza urbana; y La Tierra de Torrijos, en concentración de la propiedad rústica y urbana; todas ellas en llanura, por el contrario, un importante conjunto de comarcas periféricas de sierra y piedemonte no destacan en ninguna de las variables estudiadas, lo que marca una profunda dualidad regional.

\section{BIBLIOGRAFÍA CITADA}

CAMPOS ROMERO, M $M^{a}$ L. (1996): "La ciudad de Toledo y el Tajo: más que una singular fachada fluvial en la península rocosa", en AA.VV. II Jornadas de Geografía Urbana, Alicante, Universidad de Alicante, pp. 379-390.

CAMPOS, M ${ }^{a}$ L., ESCUDERO, L. A. y RODRIGUEZ, Ma A. (2010): "Los nuevos desarrollos urbanos en la capital de Castilla-La Mancha”, en CEBRIÁN, F. PILLET, F. y CARPIO, J. (edits) Las escalas de la Geografia: del mundo al lugar. Homenaje al profesor Miguel Panadero Moya, Cuenca, Universidad de Castilla-La Mancha, pp. 329-356.

CAÑIZARES RUIZ, Ma C (1998): Desarrollo urbano y problemática ambiental de la ciudad de Puertollano (Ciudad Real), Cuenca, Universidad de Castilla-La Mancha.

(2009): "La investigación geográfica en la Universidad de Castilla-La Mancha", en Anales de Geografía de la Universidad Complutense, vol 29, nº 1, pp. 233-253.

CAPEL, H (2007): "El debate sobre la construcción de la ciudad y el modelo Barcelona", en Scripta Nova. Revista electrónica de geografía y ciencias sociales, vol. XI, n ${ }^{\circ}$ 233, 15 de febrero

CERRO MALAGÓN, R. J. del (1988): "Desamortización y urbanismo. Estructura de Toledo en el siglo XIX”, en Toledo ¿ciudad viva? ¿ciudad muerta?, Toledo, Colegio Universitario de Toledo, pp. 369-389.

CORONADO, J., M. PILLET, F. y UREÑA, J. M. (2004): “Urbanismo y ordenación del territorio en Castilla-La Mancha", en PEDREGAL. J. M. Obras públicas en CastillaLa Mancha, Madrid, Ministerio de Fomento, pp. 193-231.

DONÉZAR DÍEZ DE ULZURRUM, J. M. (1984): Riqueza y propiedad en la Castilla del Antiguo Régimen. Madrid, Ministerio de Agricultura Pesca y Alimentación.

ESPEJO MARIN, C. (1990): "El Registro de la Propiedad expropiable en la provincia de Albacete (1933)", en Al-Basit. Revista de Estudios Albacetenses, nº 26, pp. 81-108. 
(1991): "Notas sobre la gran propiedad agraria albacetense en los años treinta", en Revista de Estudios Regionales, $\mathrm{n}^{\mathrm{o}}$ 30, pp. 137-154.

ESPEJO, C. y LÓPEZ DE LOS MOZOS, A. (1989): "Las grandes explotaciones agrarias de la provincia de Albacete", en Al-Basit Revista de Estudios Albacetenses, n 25, pp. 165-179.

FEIJOO GÓMEZ, A. (1990): La desamortización del siglo XIX en Castilla-La Mancha, Talavera de la Reina, Junta de Comunidades de Castilla-La Mancha.

FEO PARRONDO, F. (2001): "Propiedad rústica en la España interior desde una perspectiva de género", en Anales de Geografía de la Universidad Complutense, $\mathrm{n}^{\mathrm{o}}$ 21, pp. 203-221.

(2003): "Propiedad rústica en el norte de España desde una perspectiva de género", en Estudios Geográficos, n 251, págs. 221-241.

(2005) "Aproximación al análisis de la distribución de la propiedad rústica en Aragón desde una perspectiva de género", en Geographicalia, nº 47, págs. 69-86.

(2008): "Propiedad rústica en el nordeste de Castilla y León desde una perspectiva de género", en Ciencias Sociais e Humanidades, n 20, págs. 113-132.

GARCÍA BADELL, G. (1946): "Estudio sobre la distribución de la extensión superficial y de la riqueza de la propiedad agrícola en España entre las diferentes categorías de fincas", en Estudios Geográficos, n 23, págs. 171-221.

GARCÍA BALLESTEROS, A. (1978): Geografia Urbana de Guadalajara, Madrid, Fundación Universitaria Española.

GARCÍA BALLESTEROS, A. y REDONDO, A. (1984): "El papel de la desamortización en la evolución de las ciudades españolas. Los ejemplos de Guadalajara y Plasencia”, en Estudios Geográficos, n 172-173, pp. 523-544.

GARCÍA, F. y GÓMEZ, C. J. (2010): Tierra y sociedad rural en Castilla-La Mancha a finales del Antiguo Régimen, en VALLE, A. R. del (coord) Historia agraria de Castilla-La Mancha. Siglos XIX-XXI, Ciudad Real, Biblioteca Añil, pp. 83-116.

GARCÍA MARTÍNEZ, C (1995): Actividad comercial y espacio urbano: la organización espacial del comercio minorista en la ciudad de Albacete. Cuenca, Universidad de Castilla-La Mancha.

GARCÍA MARTÍNEZ, C y VAZQUEZ, A. (2007): "Las ciudades", en PILLET, F. (coord.) Geografía de Castilla-La Mancha, Ciudad Real, Almud, Ediciones de Castilla-La Mancha, pp. 159-176.

GARCÍA ROLDÁN, A. (1992): El crecimiento reciente de Guadalajara (1960-1990): implicaciones espaciales y sociales, Madrid Universidad Complutense.

GESTEIRO ARÁUJO, M. (1990): "Geografía Agraria de la Mancha Baja (Cuenca)", (Tesis doctoral) Madrid, Departamento de Geografía, Universidad Autónoma de Madrid.

(1996): "La propiedad de la tierra en La Mancha Baja (Cuenca)", en Estudios Geográficos, $n^{\circ} 225$, pp. 645-665.

GOMEZ MENDOZA, J. (1977): Agricultura y expansión urbana. La campiña del bajo Henares en la aglomeración de Madrid. Madrid, Alianza editorial.

LADRÓN DE GUEVARA, Ma P. (2010): "Reformismo agrario y experiencia revolucionaria, 1931-1939”, en VALLE, A. R. del (coord) Historia agraria de Castilla-La Mancha. Siglos XIX-XXI, Ciudad Real, Biblioteca Añil, pp. 239-270. 
LÓPEZ ONTIVEROS, A. (1978): "Medio físico e historia como conformadores del latifundio andaluz", en Agricultura y Sociedad, n 9, pp. 235-255.

LOPEZ-SALAZAR PÉREZ. J. (1986): Estructuras agrarias y sociedad rural en La Mancha (ss. XVI-XVII), Ciudad Real, Instituto de Estudios Manchegos.

LOSADA AZORÍN, A. A. (2000): Caracterización y transformación de los espacios industriales periféricos durante la potsindustrialización: la ciudad de Albacete, Tesis doctoral, Universidad de Castilla-La Mancha.

MANGAS NAVAS, J. M. (1988): "Mancomunidades concejiles de montes, pastos y caza en Castilla-La Mancha", en PILLET, F. (coord) El espacio rural de Castilla-La Mancha. Ciudad Real, Diputación de Ciudad Real, tomo II, pp. 207-234.

MARTÍN MARTÍN, V. O. (2008): "Los jornaleros y la gran propiedad agraria en el Sur de España", en Anales de Geografía de la Universidad Complutense, vol. 28, 2, 137165.

MARTINEZ GARRIDO, E. (1991): "Geografía de la caza en Ciudad Real", en Agricultura y Sociedad, no 58, pp. 263-294.

(2000): La caza en la provincia de Ciudad Real: su análisis geográfico como aprovechamiento reciente, Madrid, Universidad Autónoma de Madrid.

MARTINEZ GARRIDO, E. y TORIJA, R. (2009): "Paisajes de la caza de Ciudad Real: un ensayo de clasificación", en PILLET, F, CAÑIZARES, M ${ }^{a}$ C. y RUIZ. A. R. (coord) Geografía, territorio y paisaje: el estado de la cuestión. Actas del XXI Congreso de Geógrafos Españoles, Cuenca, Universidad de Castilla-La Mancha, pp. 1161-1175.

MARTÍNEZ SÁNCHEZ-MATEOS, H. S. (2010): La estructura del transporte y sus implicaciones territoriales en Castilla-La Mancha, Toledo, Consejo Económico y Social de Castilla-La Mancha.

MAS HERNÁNDEZ, R. (2005): "El estudio de la propiedad urbana en España", en LAVASTRE, PH y MAS, R. (coords) Propiedad urbana y crecimiento de la ciudad, Madrid, Ediciones de la Universidad Autónoma de Madrid, pp. 241-263.

MATA OLMO, R (1988): "La distribución de la propiedad y de la explotación de la tierra en Castilla-La Mancha: aspectos de la situación actual y de su dinámica reciente", en PILLET, F. (coord) El espacio rural de Castilla-La Mancha. Ciudad Real, Diputación de Ciudad Real, tomo II, pp. 165-206.

MATA, R y NARANJO, J. (1996): "La Geografía Rural y el estudio de la tenencia de la tierra en España", en LÓPEZ ONTIVEROS, A y MOLINERO, F. (coord) Primer Simposium de Geógrafos Rurales Británicos y Españoles. La investigación hispanobritánica reciente en Geografia Rural: del campo tradicional a la transición postproductivista, Asociación de Geógrafos Españoles. Murcia. pp. 95-110.

MEJÍAS LÓPEZ, J. (2008): Antropología urbana de Talavera de la Reina: ciudad y territorio, Ciudad Real, Almud.

MONTEAGUDO LÓPEZ-MENCHERO, J. (2010): "Población y territorio en CastillaLa Mancha: 1900-2008. Una aproximación comarcal y municipal”, en CEBRIÁN, F. PILLET, F. y CARPIO, J. (edits) Las escalas de la Geografia: del mundo al lugar. Homenaje al profesor Miguel Panadero Moya, Cuenca, Universidad de Castilla-La Mancha, pp. 49-106.

PANADERO MOYA, M. (1976): La ciudad de Albacete. Albacete, Caja de Ahorros Provincial de Albacete. 
PANADERO, M. y PILLET, F. (1999): “Las comarcas de la región”, en TAMAMES, R. y HERAS, R. (Dirs) Enciclopedia de Castilla-La Mancha, Madrid, Edicsa 92, tomo 2, pp. 175-215.

PILLET CAPDEPÓN, F. (1984): Geografia Urbana de Ciudad Real (1255-1980), Madrid, Akal-Universitaria.

(1987): "Almagro y Ciudad Real: el pleito sobre la capitalidad", en Primera semana de Historia de Almagro, Ciudad Real, Diputación Provincial, pp. 81-97.

(1988 a): "La ciudad de Almagro: Evolución de la estructura de la propiedad urbana", en Estudios Geográficos, nº 193, pp. 657-670.

(1988 b): "La provincia de Ciudad Real en el contexto actual de las grandes explotaciones y propiedades agrarias nacionales", en PILLET, F. (coord) El espacio rural de Castilla-La Mancha, Ciudad Real, Diputación de Ciudad Real, tomo II, pp. 235.248.

(1989 a): "Los regadíos de Castilla-La Mancha: implicaciones actuales y tipos de explotación", en Los paisajes del agua. Libro jubilar dedicado al profesor Antonio López Gómez, Valencia, Universidades de Valencia y Alicante, pp. 133-143.

(1989b): "La necesaria reforma estructural en zonas desfavorecidas con predominio de latifundios de secano: la comarca de los Montes Norte de Ciudad Real", en $V$ Coloquio de Geografia Agraria, Santiago de Compostela, Universidade de Santiago de Compostela, pp. 147-155.

(1991): "Las estructuras agrarias y los usos del suelo", en PILLET, F. (coord). La provincia de Ciudad Real. Geografía, Ciudad Real, Diputación de Ciudad Real, pp. 221-272.

(1995): "El medio ambiente urbano de Ciudad Real", en Anales de Geografía de la Universidad Complutense, $\mathrm{n}^{\circ} 15$, pp. 521-537.

(2001): La Mancha. Transformaciones de un espacio rural, Madrid. Biblioteca Añil.

(2005): "Ciudad Real: Cambios en el espacio público y en la vida colectiva con la llegada del AVE a la ciudad", en GUTIERREZ, O. (coord) La ciudad y miedo. VII Coloquio de Geografia Urbana, Girona, AGE y Universidad de Girona, pp. 325-334

(2007): "Catastro y propiedad rústica y urbana (1750-2005) y su relación actual con las Comunidades Autónomas. Una aplicación a Castilla-La Mancha”, en Boletín de la Asociación de Geógrafos Españoles n ${ }^{\circ} 45$, pp. 213-232.

(2008 a): "La actual concentración de la propiedad rústica y su riqueza catastral en los municipios de España", en Investigaciones Geográficas, $\mathrm{n}^{\circ}$ 45, pp. 9-28.

(2008 b): "El catastro inmobiliario territorial de urbana y rústica en España", en Scripta Nova. Revista Electrónica de Geografía y Ciencias Sociales, vol. XII, n² 274, 1 de octubre (www.ub.es/geocrit/sn/sn-274.htm).

(2008 c): Espacio y Ciencia del territorio. Proceso y relación global-local, Madrid, Biblioteca Nueva.

(2009): "La concentración de la propiedad rústica y urbana en España y sus fuentes: escalas actuales y evolución catastral", en Ería. Revista de Geografia, nº 80, pp. 247-258.

(2010 a): "La planificación urbanística y estratégica y su relación con la valoración catastral en España", en Boletín de la Asociación de Geógrafos Españoles, n ${ }^{\circ}$ 54, pp. 323-334.

(2010 b): "La diversidad de Castilla-La Mancha: la comarcalización geográfica y sus municipios", en CEBRIÁN, F., PILLET, F, y CARPIO, J. (edits) Las escalas de la Geografia: del mundo al lugar. Homenaje al profesor Miguel Panadero Moya, Cuenca, Universidad de Castilla-La Mancha, pp. 25-48. 
PILLET, F. et al (2010): "El policentrismo en Castilla-La Mancha y su análisis a partir de la población vinculada y el crecimiento demográfico", en Scripta Nova. Revista Electrónica de Geografia y Ciencias Sociales, vol. XIV, n 321, 20 de abril.

PILlET, F. (coord) (1988): El espacio rural de Castilla-La Mancha, Ciudad Real, Diputación de Ciudad Real (2 tomos).

QUIRÓS LINARES, F. (1992): "Valle de Alcudia y Campo de Calatrava", en F. QUIRÓS y G. PLANCHUELO El paisaje geográfico. Valle de Alcudia, Campo de Calatrava y Campo de Montiel, (facsímil), Ciudad Real, Diputación Provincial, pp. 21-227.

RODRIGUEZ DOMENECH, $\mathrm{M}^{\mathrm{a}} \mathrm{A}$. (en prensa): Las transformaciones urbanas de Ciudad Real durante las últimas décadas, Ciudad Real, Instituto de Estudios Manchegos.

RODRÍGUEZ ESPINOSA, E. (2000): El espacio rural del Campo de Calatrava (Ciudad Real) en la década de los 80 (situación inmediata al ingreso de España en la Unión Europea), Ciudad Real, Instituto de Estudios Manchegos.

RODRÍGUEZ RODRIGUEZ, V. (1984): La tierra en la Sagra toledana: su evolución de los siglos XVI a XX, Toledo, Caja de Ahorros Provincial de Toledo.

ROMERO GONZÁLEZ, J. (1983): Propiedad agraria y sociedad rural en la España mediterránea. Los casos valenciano y castellano en los siglos XIX y XX. Madrid. Ministerio de Agricultura, Pesca y Alimentación.

RUIZ PULPÓN, A. R. (2007): Tipología territorial de la agricultura de regadio en los municipios de la cuenca hidrográfica del Guadiana, Toledo, Consejo Económico y Social de Castilla-La Mancha.

SANTOS SANTOS, J. F. (2010): "Diferencias territoriales en los Montes de Toledo (Ciudad Real): Estados del Duque y Cabañeros", en CEBRIÁN, F., PILLET, F, y CARPIO, J. (edits) Las escalas de la Geografía: del mundo al lugar. Homenaje al profesor Miguel Panadero Moya, Cuenca, Universidad de Castilla-La Mancha, pp. 279-301.

SANZ JARQUE, J .J. (1975): "La cuestión de la tierra en los Estados del Duque", en CASTRO, F. (coord) Estudios jurídicos en homenaje al profesor Federico Castro, Madrid, Instituto Nacional de Estudios Jurídicos-Tecnos, pp. 641-702.

SIMÓN SEGURA, F. (1974): "La desamortización de 1855 en la provincia de Ciudad Real", en Revista de Hacienda Pública, no 27, pp. 87-114.

TATJER MIR, M. (1988a): "La Contribución Territorial Urbana (1716-1906)", en SEGURA, A. y CANET, I. (coords) El Catastro en España 1714-1906, Madrid, Ministerio de Economía y Hacienda, vol-I, pp. 135-173.

(1988b): "La Contribución Territorial Urbana a lo largo del siglo XX (1906-1979)", en SEGURA, A. y CANET, I. (coords) El Catastro en España 1906-1989, Madrid. Ministerio de Economía y Hacienda. vol-II, pp. 61-101.

TROITIÑO VINUESA, M. A. (1984): Cuenca. Evolución y crisis de una vieja ciudad castellana. Madrid. Universidad Complutense de Madrid.

(1998): "Paisaje urbano y patrimonio cultural: el centro histórico de Cuenca", en MARTINEZ DE PISÓN, E. (coord) Paisaje y Medio Ambiente, Valladolid, Universidad de Valladolid, pp. 117-136.

VALLE, A. R. del (coord) (2010): Historia agraria de Castilla-La Mancha. Siglos XIX$X X I$, Ciudad Real, Biblioteca Añil. 
VARONA ALABERN, J. E. (2001): El Valor Catastral: su gestión e impugnación. Análisis jurídico de la gestión catastral y de la gestión tributaria en el Impuesto sobre Bienes Inmuebles, Editorial Aranzadi, Aoiz.

VAZQUEZ GONZÁLEZ, A. (1988): "La concentración parcelaria: Elemento de transformación del espacio rural de Castilla-La Mancha", en PILLET, F. (coord) El espacio rural de Castilla-La Mancha, Ciudad Real, Diputación de Ciudad Real, tomo II, pp. 43-59.

ZARATE MARTÍN, M. A. (1984): “Análisis de la dotación y jerarquización funcional del espacio urbano de Toledo mediante el uso de técnicas cuantitativas", en Estudios Geográficos, $\mathrm{n}^{\mathrm{o}}$ 176, pp. 329-352.

(2000): "Toledo, Ciudad Patrimonio de la Humanidad", en Boletín de la Real Sociedad Geográfica, tomo CXXXVI, pp. 137-184.

(2007): "Toledo. Planeamiento y especulación en ciudades históricas", en Anales de Geografia de la Universidad Complutense, vol, 27, n² 2, pp. 151-175.

(2010): "Paisajes culturales urbanos, un legado para conservar", en Anales de Geografía de la Universidad Complutense, vol, 30, $\mathrm{n}^{\circ}$ 2, pp. 187-210. 
Anexo 1

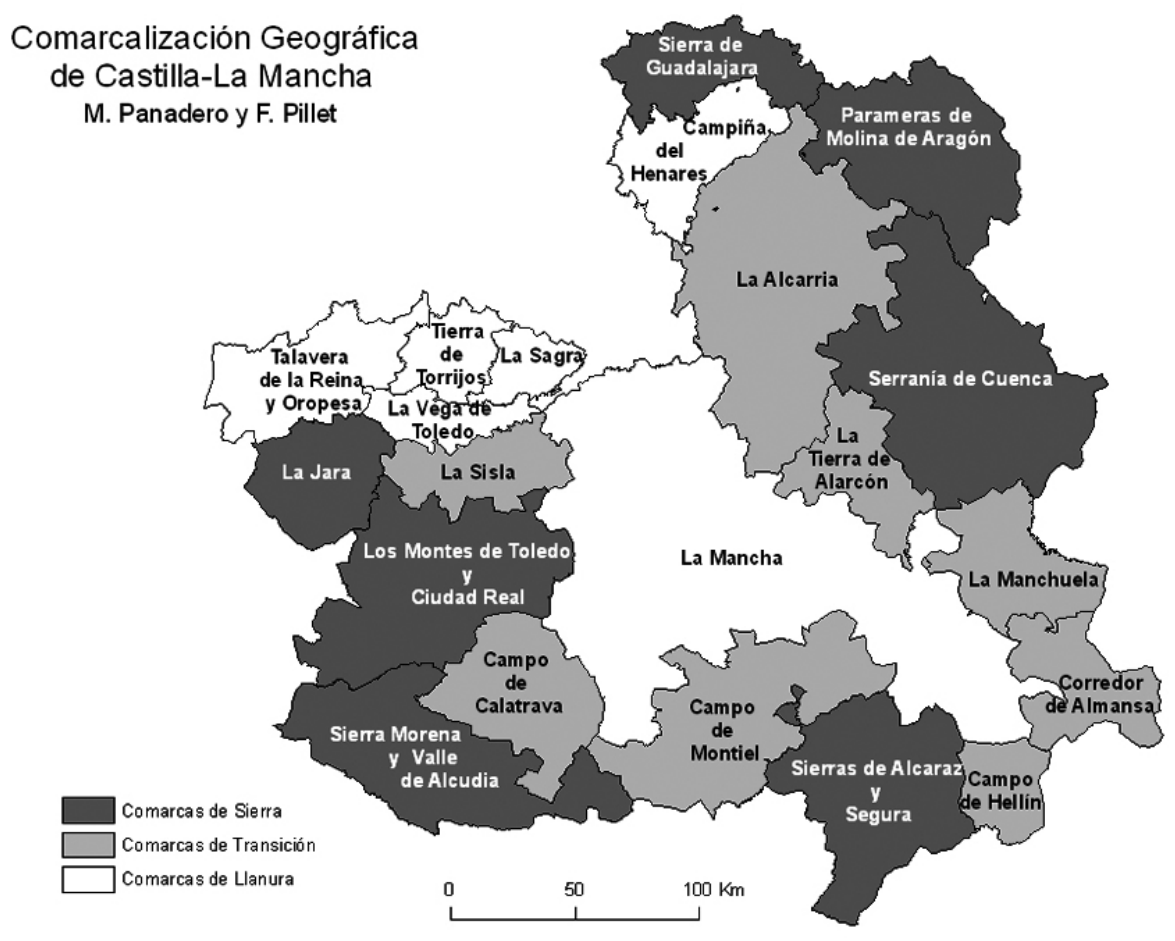


Anexo 2. Valoración Catastral Rústica y de Urbana en las comarcas de Castilla-La Mancha: concentración de la propiedad y riqueza

\begin{tabular}{|l|c|c|c|c|}
\hline \multirow{2}{*}{ TIPOS DE COMARCAS } & \multicolumn{2}{|c|}{ RÚSTICA } & \multicolumn{2}{c|}{ URBANA } \\
\cline { 2 - 5 } & C. Propiedad & Riqueza Catastral & C. Propiedad & Riqueza Catastral \\
\hline DE LLANURA & VC/T & VC/S & VC/T & VC/S \\
\hline La Mancha & & & & \\
\hline Tierra de Torrijos & 5,4 & 0,7 & 0,06 & 0,05 \\
\hline Vega de Toledo & 7,7 & 0,5 & 0,06 & 0,03 \\
\hline La Sagra & 8,9 & 0,8 & 0,06 & 0,04 \\
\hline Talavera de la Reina & 4,6 & 0,7 & 0,08 & 0,03 \\
\hline Campiña del Henares & 3,6 & 0,4 & 0,05 & 0,03 \\
\hline -Media comarcal & 2,1 & 0,3 & 0,04 & 0,04 \\
\hline DE TRANSICIÓN & 5,4 & 0,6 & 0,06 & 0,04 \\
\hline La Alcarria & 1,8 & 0,2 & 0,03 & 0,03 \\
\hline Tierra de Alarcón & 2,7 & 0,3 & 0,02 & 0,03 \\
\hline La Manchuela & 2,5 & 0,4 & 0,04 & 0,05 \\
\hline Corredor de Almansa & 4,5 & 0,4 & 0.04 & 0,04 \\
\hline Campo de Hellín & 4,4 & 0,5 & 0,04 & 0,04 \\
\hline Campo de Montiel & 3,7 & 0,3 & 0,04 & 0,04 \\
\hline Campo de Calatrava & 6,3 & 0,4 & 0,05 & 0,04 \\
\hline La Sisla & 2,9 & 0,4 & 0,06 & 0,03 \\
\hline -Media comarcal & 3,6 & 0,4 & 0,04 & 0,04 \\
\hline DE SIERRA & & & & \\
\hline Sierra de Guadalajara & 0,8 & 0,1 & 0,01 & 0,02 \\
\hline Molina de Aragón & 2,3 & 0,1 & 0,02 & 0,03 \\
\hline Serranía de Cuenca & 2,0 & 0,1 & 0,02 & 0,03 \\
\hline Sierras de Alcaraz y S. & 2,1 & 0,2 & 0,03 & 0,04 \\
\hline Sierra Morena y V. A. & 4,1 & 0,2 & 0,04 & 0,04 \\
\hline Los Montes de T - CR & 4,1 & 0,2 & 0,04 & 0,04 \\
\hline La Jara & 2,2 & 0,2 & 0,03 & 0,03 \\
\hline -Media comarcal & 2,5 & 0,1 & 0,03 & 0,03 \\
\hline MEDIA REGIONAL & 3,4 & 0,4 & 0,04 & 0,04 \\
\hline & & & & \\
\hline & & & & \\
\hline
\end{tabular}

Fuente: Dirección General del Catastro, 2005 (Elaboración propia) 